\title{
Urine Neuron-Specific Enolase and Its Clinical Implication in Patients with Neuroblastoma
}

\author{
Үoн-Ichi Gotoh, Kumiko Нashimoto and Keiya Tada \\ Department of Pediatrics, Tohoku University School of \\ Medicine, Sendai 980
}

\begin{abstract}
Gотон, Y-I., Наsнimoto, K. and Tada, K. Urine Neuron-Specific Enolase and Its Clinical Implication in Patients with Neuroblastoma. Tohoku J. exp. Med., 1986, 149 (1), 67-72 — Urine levels of neuron-specific enolase (NSE) were determined in 6 patients with neuroblastoma, in 72 controls and in 5 infants with hematuria by means of a double-antibody inhibition radioimmunoassay method. Urine levels (NSE $\mathrm{ng}$ /creatinine $\mathrm{mg}$ ) in 2 patients with advanced neuroblastoma were elevated (3.03 \pm 0.28 (S.D.)), when compared with those of 4 patients with neuroblastoma in remission $(0.65 \pm 0.26$ (s.D. $))$, 10 healthy neonates $(1.26 \pm 0.42$ (S.D.)), 25 healthy infants $(0.51 \pm 0.26$ (s.D. $))$, and 37 healthy adults $(0.37 \pm 0.17$ (S.D.)). Urine levels in 4 infants with microhematuria and an infant with macrohematuria were $1.62 \pm 0.10$ (s.D.) and 33.83, respectively. Serial measurements in 3 patients with neuroblastoma receiving various therapies have revealed that there was a good correlation between urine NSE level and the response to therapy. These results indicate that NSE in urine may be a valuable marker for monitoring the effectiveness of therapy in patients with neuroblastoma.—urine; neuron-specific enolase; neuroblastoma.
\end{abstract}

NSE or $\gamma$-enolase was originally extracted from the brain and was later characterized as a specific neuronal isomer of the widely distributed glycolytic enzyme, 2-phosphoglycerate hydrolase (Moore and McGregor 1965; Fletcher et al. 1976). Enolase is a dimer composed of three immunologically distinct subunits, $\alpha, \beta, \gamma$.

Recently, it has been reported that a large amount of NSE was produced in neuroendocrine tumors (Tapia et al. 1982). Significantly raised levels of serum NSE were seen in patients with neuroblastoma. These results raise the possibility of serum NSE measurement as an additional and simple diagnostic method (Ishiguro et al. 1982, 1983). We also reported that urine levels of NSE were higher in an infant with advanced neuroblastoma than those in controls (Gotoh et al. 1985). In this paper, we examined the correlation between urine NSE levels and clinical features in 3 patients with neuroblastoma in details.

Received December 26, 1985 ; accepted for publication April 17, 1986. 


\section{Materials and Methods}

\section{Subjects}

Control samples consisted of urines from 10 healthy neonates, 25 healthy infants, 37 healthy adults, 4 infants with microscopic hematuria and an infant with macroscopic hematuria. Urine samples were obtained from 6 patients with neuroblastoma, including 4 patients in remission, a patient in the advanced state and a patient who was newly diagnosed. The patients' status was staged according to the criteria of Evans (1980). The clinical data for 3 patients in whom serial measurements of urine NSE were done, were shown in Table 1 .

\section{Sampling procedures}

The samples were removed as soon as possible after the passage of urine, added with $50 \%$ of glycerol in final $10 \%$ glycerol concentration, and then stored at $-4{ }^{\circ} \mathrm{C}$ until assayed. Previous studies had shown that urine NSE level was maintained for at least one week after sampling (data not shown). All of the samples were processed within one week.

\section{NSE assay}

We used a commercial double-antibody RIA Kit (NSE Kit, Eiken Immunochemical Laboratory, Tokyo) to determine NSE concentrations by a modification of the previously described method (Notomi et al. 1985). Urine samples were assayed in duplicate. In brief, NSE in the samples or standard solutions was incubated with ${ }^{125}$ I-labelled NSE and the rabbit antibody specific to the $\gamma$-subunit of human enolase at room temperature for $24 \mathrm{hr}$ in tubes. Antigen-antibody complexes were incubated with the goat anti-rabbit IgG antibody as a secondary antibody at room temperature for $1 \mathrm{hr}$. The precipitate was centrifuged at $4,300 \times \mathrm{g}$ at $4^{\circ} \mathrm{C}$ for $1 \mathrm{hr}$ and after centrifugation, the radioactivity of the pellet was counted with a well-type gamma counter.

\section{Creatinine assay}

We used a commercial kit (Creatinine-Test Wako, Wako Pure Chemicals Ltd., Tokyo) to determine urine creatinine $(\mathrm{Cr})$ by a spectrophotometric method.

\section{Statistical analysis}

Values are given in terms of mean \pm S.D.. Statistical significance of differences between mean values of controls and advanced neuroblastomas was evaluated by Student's $t$-test. Differences were judged to be significant when $p$ values were less than 0.05 .

\section{RESULTS}

The clinical data and serum NSE levels in three patients with neuroblastoma are shown in Table 1. All of them showed high levels of serum NSE on admission. Levels of urinary catecholamine metabolites in patients were variable; in Case 1, HVA was high, but VMA was within normal range; in Case 2, HVA was slightly elevated, and in Case 3 , both HVA and VMA were elevated.

The mean values of urine NSE (ng/Cr mg) are shown in Table $2 ; 0.51 \pm 0.26$ in control infants, $1.26 \pm 0.42$ in control neonates, $0.36 \pm 0.17$ in control adults, $1.62 \pm 0.40$ in the infants with microscopic hematuria, 33.83 in an infant with macroscopic hematuria, $3.03 \pm 0.28$ in two patients with advanced neuroblastoma, $0.65 \pm 0.26$ in four patients with neuroblastoma in remission. 
TABle 1. Serum NSE levels and clinical data for 3 patients with neuroblastoma on admission

\begin{tabular}{|c|c|c|c|c|c|c|c|}
\hline \multirow[b]{2}{*}{ Case } & \multirow[b]{2}{*}{ Age } & \multirow[b]{2}{*}{ Sex } & \multirow[b]{2}{*}{ Histology } & \multirow[b]{2}{*}{$\begin{array}{l}\text { Disease } \\
\text { state }\end{array}$} & \multirow{2}{*}{$\begin{array}{l}\text { Serum } \\
\mathrm{NSE} \\
(\mathrm{ng} / \mathrm{ml})\end{array}$} & \multicolumn{2}{|c|}{ Urine } \\
\hline & & & & & & $\underset{(\mathrm{m}}{\mathrm{VMA}}$ & $\begin{array}{l}\text { HVA } \\
\text { ay) }\end{array}$ \\
\hline 1 & $2 y 7 m$ & M & NB & IV B & 128.0 & 5.8 & 26.6 \\
\hline 2 & $25 \mathrm{~d}$ & M & N.T. & IV S & 26.0 & 7.5 & 11.0 \\
\hline 3 & $1 \mathrm{y} 4 \mathrm{~m}$ & M & GNB & IV A & 35.5 & 11.8 & 19.0 \\
\hline
\end{tabular}

GNB, ganglioneuroblastoma; HVA, homovanillic acid; NB, neuroblastoma; N.T., not tested; VMA, vanillyl-manderic acid.

$\mathrm{Y}$, year ; m, month; d, day.

TABLE 2. Urine NSE levels in patients with neuroblastoma and controls

\begin{tabular}{lcc}
\hline & $\mathrm{n}$ & Urine NSE (ng/cr mg) \\
\hline Healthy neonates & 10 & $1.26 \pm 0.42$ \\
Healthy infants & 25 & $0.51 \pm 0.26$ \\
Healthy adults & 37 & $0.36 \pm 0.17$ \\
Infants with microhematuria & 4 & $1.62 \pm 0.40$ \\
Infant with macrohematuria & 1 & 33.83 \\
Neuroblastoma & 2 & $3.03 \pm 0.28$ \\
Neuroblastoma in remission & 4 & $0.65 \pm 0.26$
\end{tabular}

cr, creatinine. Values are means \pm s.D.

Serial measurements of urine NSE levels were performed in Case 1 (an advanced state), Case 2 (a newly diagnosed state), and Case 3 (a remission state) during the course of treatment, as shown in Figs. 1, 2 and 3, respectively. As shown in Fig. 1, serum NSE levels gradually increased as the patient's condition deteriorated. Clinically this patient failed to respond to chemotherapy. Urine NSE levels increased transiently during chemotherapy. After chemotherapy, urine NSE levels decreased to the pre-treatment level. The more effective the treatment was, the higher the urine NSE level during treatment.

As shown in Case 2, serum NSE levels decreased temporarily to nearly normal level when the patient responded to the chemotherapy and/or radiotherapy. Urine NSE levels also gradually decreased as the patient responded to the treatment.

As shown in Case 3, urine NSE levels did not significantly increase during the consolidation treatment in remission. Serum NSE levels remained within normal range.

\section{Discussion}

We described the clinical implication with regard to the therapy which might 


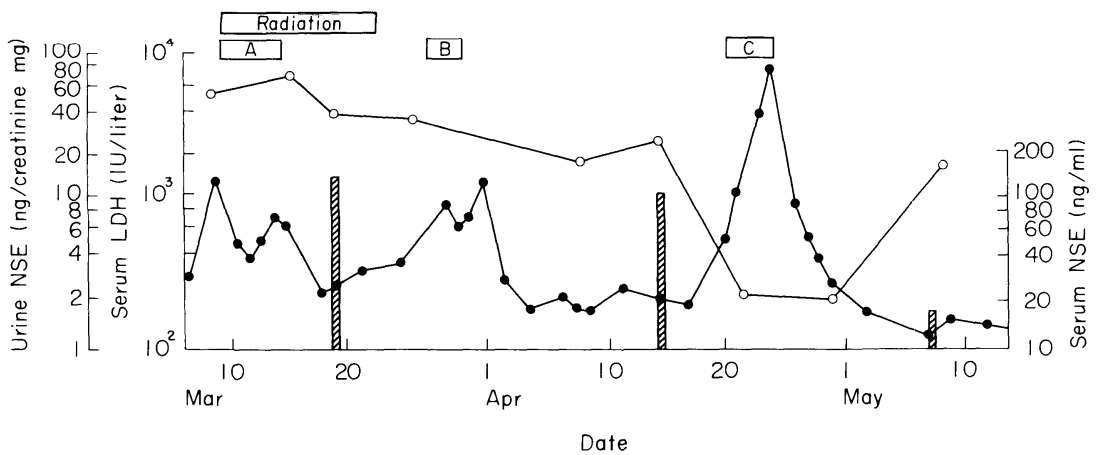

Fig. 1. Serial measurements of serum LDH, serum NSE and urine NSE levels in Case 1. —— urine NSE (ng/cr mg); ○ - • VIIIA, serum NSE $(\mathrm{ng} / \mathrm{ml})$. Radiation: 2,000 rad (metastatic retroorbital tumor)
A Dacarbazine 5 days
Cyclophosphamide 2 days
Vincristine 1 day
B Cisplatin 1 day
Teniposide 1 day
C Cyclophosphamide 7 days
Doxorubicin 1 day

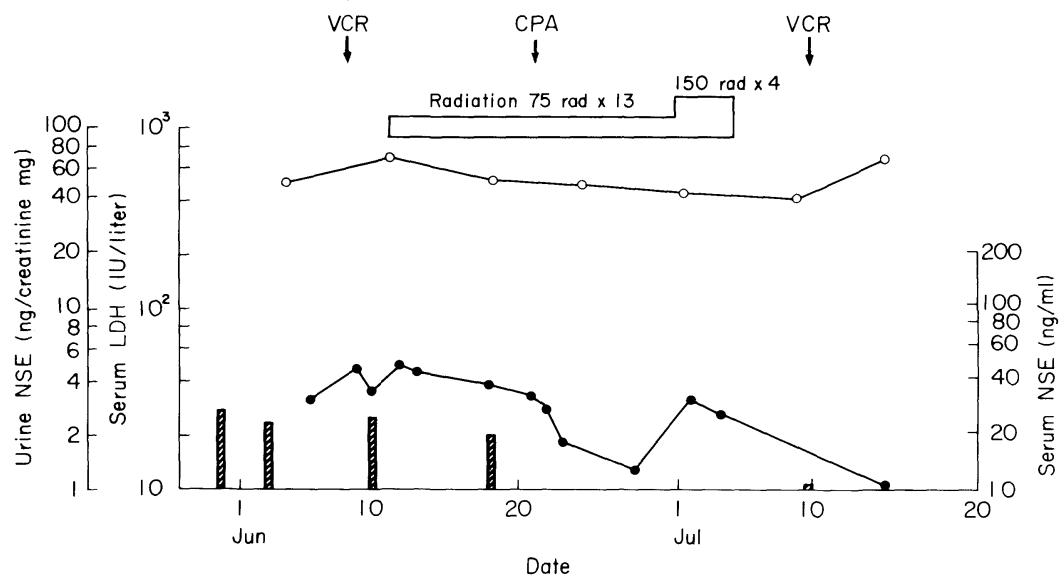

Fig. 2. Serial measurements of serum LDH, serum NSE and urine NSE levels in Case 2. VIIII, serum NSE $(\mathrm{ng} / \mathrm{ml})$.

Radiation: 1,175 rad (primary hepatic tumor)

VCR: Vincristine 1 day

CPA : Cyclophosphamide 1 day

bring about the release of a large amount of NSE into the urine. Tapia et al. reported that neuroendocrine tumors produce a considerable amount of NSE, and suggested that the measurement of serum NSE might be a diagnostic test for neuroblastoma. Our report was one of the interesting matters which described a novel approach to monitor neuroblastoma activity in vivo. 


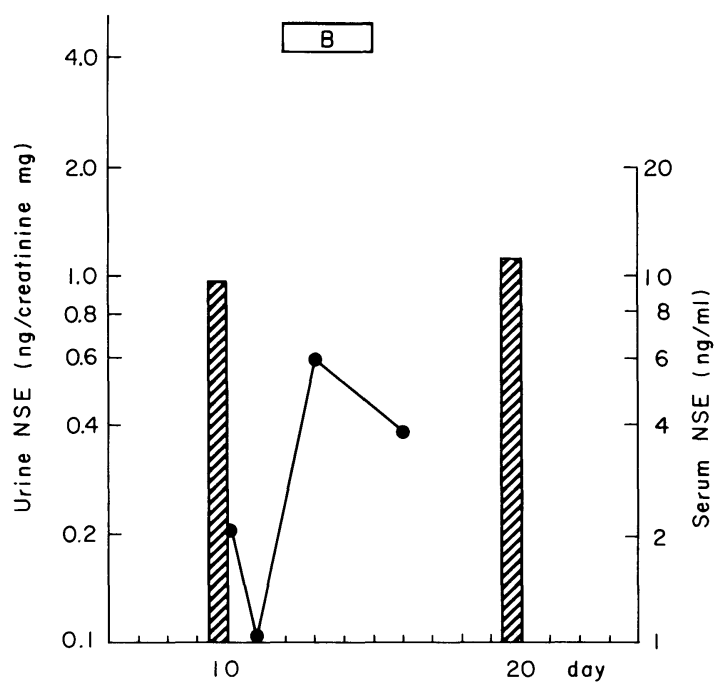

Fig. 3. Serial measurements of serum NSE and urine NSE levels in Case 3.

-——, urine NSE (ng/cr mg); VIIIIA, serum NSE (ng/ml)

B : Cisplatin 1 day

Teniposide 1 day

NSE seems to be an intracellular enzyme and only a small proportion of it is extracellularly released (unpublished data). There could be a problem with the externalization of this enzyme and that this would create the difficulty in using NSE as an indicator for the number of tumor cells in vivo.

We could examine what would happen if cells were killed and lysed by chemotherapy or radiotherapy in vivo, using serial measurements of urine NSE levels in patients with neuroblastoma. Sequential analysis of urine NSE levels in three clinical states (an advanced state, a newly diagnosed state, and a remission state) revealed that increased levels of NSE in the urine were probably due to the destruction of tumor cells. The high level of NSE in the urine possibly indicates that the patient has an appreciable size of tumor mass, from which NSE is leaking into the bloodstream and then excreting into the urine, due to cytotoxic effects. The lower level of NSE in the urine after effective or ineffective therapy may be due to a decrease of NSE leaking into the bloodstream and excreting into the urine from the tumor mass. These results suggest that NSE levels are not dependent on the number of the tumor cells which are either in the proliferating state or in the non-proliferating state, but on the number of the tumor cells which are lysed. Therefore, our present study on serum and/or urine NSE in patients with neuroblastoma indicates that the measurement of urine NSE level is more useful in assessing the severity of the disease and the response to the therapy than in making a diagnosis. 


\section{Acknowledgments}

This investigation was supported in part by grants from the Ministry of Education, Science and Culture.

\section{References}

1) Evans, A.E. (1980) Staging and treatment of neuroblastoma. Cancer, 45, 17991802.

2) Fletcher, L., Rider, C.C. \& Taylor, C.B. (1976) Enolase isoenzymes. III. Chromatographic and immunological characteristics of rat brain enolase. Biochim. biophys. Acta, 452, 245-252.

3) Gotoh, Y-I., Hashimoto, K. \& Tada, K. (1985) Determination of urine neuron-specific enolase levels in an advanced neuroblastoma and two neuroblastoma patients. Tohoku J. exp. Med., 147, 429-430.

4) Ishiguro, Y., Kato, K., Shimizu, A., Ito, T. \& Nagaya, M. (1982) High levels of immunoreactive nervous system-specific enolase in sera of patients with neuroblastoma. Clin. chim. Acta, 121, 173-180.

5) Ishiguro, Y., Kato, K., Ito, T., Nagaya, M., Yamada, N. \& Sugitoh, T. (1983) Nervous system-specific enolase in serum as a marker for neuroblastoma. Pediatrics, 3, 696700 .

6) Moore, B.W. \& McGregor, D. (1965) Chromatographic and electrophoretic fractionation of soluble protein of brain and liver. J. biol. Chem., 240, 1647-1653.

7) Notomi, T., Morikawa, J., Kato, K., Tsuchida, Y. \& Ohsawa, R. (1985) Radioimmunoassay development for human neuron-specific enolase : With some clinical results in lung cancers and neuroblastoma. Tumor Biol., 6, 57-66.

8) Tapia, F.J., Polak, J.M., Barbosa, A.J.A., Bloom, S.R., Marangos, P.J., Dermody, C. $\&$ Pearse, A.G.E. (1982) Neuron-specific enolase is produced by neuroendocrine tumors. Lancet, 1, 1126. 